\title{
A Study of Mallory-Weiss Syndrome Secondary to Upper Gastrointestinal Bleeding
}

\author{
Yoshihiro Inoue*, Yasuhisa Fujino, Makoto Onodera, Satoshi Kikuchi, Masayuki Sato, \\ Hisaho Sato, Hironobu Noda, Masahiro Kojika, Yasushi Suzuki, Shigeatsu Endo \\ Department of Critical Care Medicine, School of Medicine, Iwate Medical University, Morioka-shi, Japan

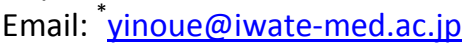

Received 4 June 2014; revised 4 July 2014; accepted 4 August 2014

Copyright (C 2014 by authors and Scientific Research Publishing Inc.

This work is licensed under the Creative Commons Attribution International License (CC BY).

http://creativecommons.org/licenses/by/4.0/

c) (7) Open Access

\begin{abstract}
Background: In Mallory-Weiss Syndrome (MWS), vomiting causes the mucous membrane and submucosa near the esophagogastric mucosal junction to tear and bleed. Thus, MWS can arise after heavy drinking and as a complication of endoscopic upper gastrointestinal examinations or procedures. However, there has been no report of MWS secondary to upper gastrointestinal bleeding thus far. Case Subjects: Of 79 MWS cases over a 10-year period from 2002 to 2011, we identified and studied 6 cases, in which MWS was probably caused by another lesion causing upper gastrointestinal bleeding. Results: There were 2 cases, each of gastric ulcers, duodenal ulcers, and varicose veins. In 3 cases, MWS was discovered during treatment of the primary lesion and was simultaneously treated. In the other 3 cases, patients were treated for MWS according to endoscopic diagnosis, and the primary lesion was overlooked; in these cases, the primary lesion was identified and treated after the bleeding recurred. One patient with cirrhosis died of hepatic failure. Conclusion: Care should be taken when dealing with MWS because it can occur as a result of vomiting caused by hematemesis or as a complication of endoscopic examination and treatment. When assessing MWS, other hemorrhagic lesions may be overlooked.
\end{abstract}

\section{Keywords}

Mallory-Weiss Syndrome, Upper Gastrointestinal Bleeding, Endoscopic Hemostasis

\section{Introduction}

Mallory-Weiss syndrome (MWS) is characterized by lacerations at the oral or anal side of the esophagogastric

"Corresponding author.

How to cite this paper: Inoue, Y., Fujino, Y., Onodera, M., Kikuchi, S., Sato, M., Sato, H., Noda, H., Kojika, M., Suzuki, Y. and Endo, S. (2014) A Study of Mallory-Weiss Syndrome Secondary to Upper Gastrointestinal Bleeding. Open Journal of Clinical Diagnostics, 4, 130-136. http://dx.doi.org/10.4236/ojcd.2014.43021 
mucosal junction in response to frequent vomiting or retching. It is a major cause of upper gastrointestinal bleeding and is traditionally associated with heavy alcohol consumption. However, MWS is increasingly caused by other factors. Because the cardinal symptom of upper gastrointestinal bleeding is hematemesis, this can sometimes lead to MWS. In some cases, MWS may develop secondary to another upper gastrointestinal bleed. Here we report several cases of MWS that occurred in such a scenario.

\section{Participants}

We identified 79 cases of MWS that were examined at our facility during a 10-year period from 2002 to 2011. 33 cases of them were treated by endoscopic hemostasis.2 cases bled again and were treated endoscopy successfully. Only one case who suffer from liver cirrhosis died of hepatic disorder. The primary lesion was related to upper gastrointestinal bleeding in 6 patients (4 men and 2 women) aged 54 - 72 years (mean age, 61.2 years). At our facility, endoscopic hemostasis was achieved using a heater probe for bleeding gastric ulcers, duodenal ulcers, and MWS, and emergency ethanolamine oleate (EO) sclerotherapy was used for ruptured varices.

\section{Results}

There were 2 cases each of gastric ulcers, duodenal ulcers, and ruptured varices among the 6 identified cases. In 3 cases, MWS was identified during treatment for the primary lesion and was simultaneously treated. In the remaining 3 cases, only MWS was diagnosed at initial emergency endoscopy, and the primary lesion was overlooked. In these cases, the primary lesion was subsequently observed and treated when the bleeding recurred a few days later. Hemostasis was achieved for both the primary lesion and MWS in all cases, but one patient died of prolonged shock from a ruptured varicose vein. Each case is outlined below.

\subsection{Bleeding Gastric Ulcers}

Case 1: A 58-year-old woman. Emergency endoscopy was performed because of hematemesis, which revealed an ulcer in the greater curvature of the antrum of the stomach with an exposed vessel. A hemostatic procedure was performed and subsequent observation revealed MWS. Therefore, another hemostatic procedure was performed at that site, and the patient was hospitalized for treatment. She recovered well and did not experience recurrent bleeding (Figure 1).

Case 2: A 64-year-old man with a history of cirrhosis. An emergency endoscopy performed for hematemesis revealed MWS, and a hemostatic procedure was performed. The patient was hospitalized for treatment, and after the bleeding recurred 3 days later, emergency endoscopy was repeated. This revealed that the antrum of the stomach was deformed and that an ulcer was overlooked. A hemostatic procedure was performed to treat the ulcer, and there was no recurrent bleeding (Figure 2).

\subsection{Bleeding Duodenal Ulcers}

Case 3: A 62-year-old man. An emergency endoscopy performed for hematemesis revealed MWS, and a hemostatic procedure was performed. The patient was hospitalized for treatment, and after the bleeding recurred 2 days later, emergency endoscopy was repeated. This revealed that an ulcer directly below the superior duodenal angle that was overlooked. A hemostatic procedure was performed, and there was no recurrent bleeding (Figure 3).

Case 4: A 72-year-old woman with a history of cerebral infarction. Emergency endoscopy was performed because of hematemesis and revealed bleeding from an ulcer directly below the superior duodenal angle. A hemostatic procedure was performed, and MWS was revealed during observation, which necessitated another hemostatic procedure. The patient was hospitalized, had no recurrent bleeding, and recovered well (Figure 4).

\subsection{Bleeding Varices}

Case 5: A 54-year-old man was under observation for cirrhosis (Child-Pugh score C). After emergency endoscopy for hematemesis revealed MWS, a hemostatic procedure was performed and the patient was hospitalized. The bleeding recurred the following day, and emergency endoscopy revealed that a varicose vein at the esophagogastric mucosal junction had ruptured. Sclerotherapy was performed, but the patient died of hepatic failure after 3 days (Figure 5). 


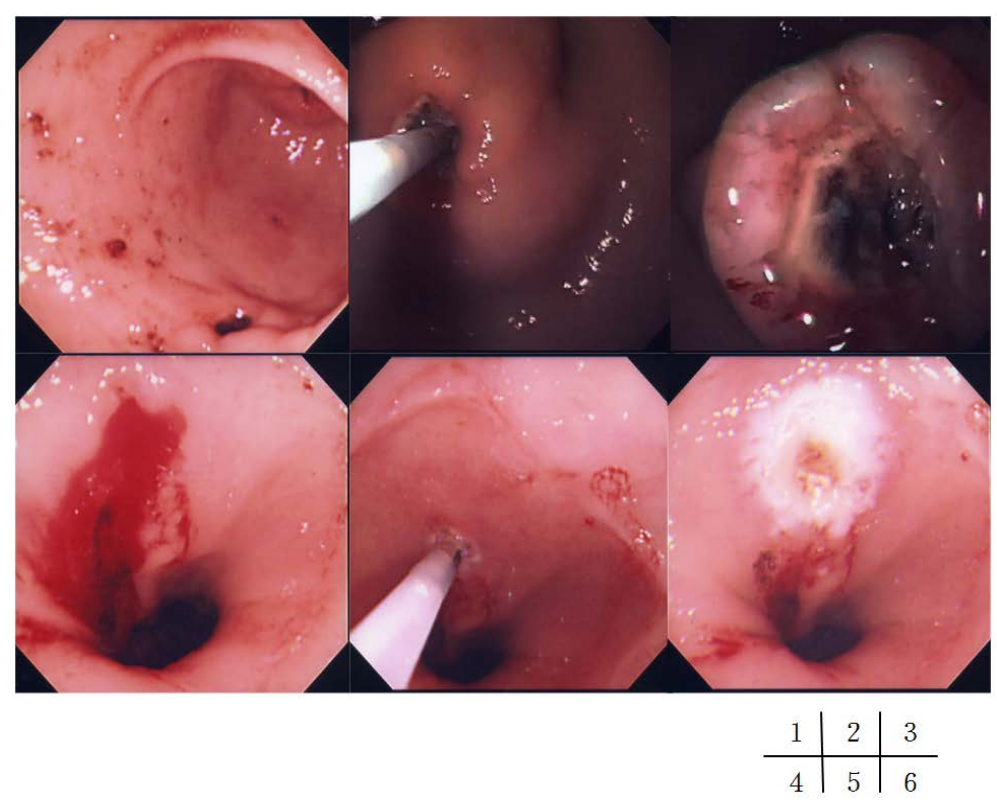

Figure 1. Upper gastrointestinal endoscopy of the case 1. 1: Ulcer of gastric antrum; 2: Hemostasis with Heater probe was performed to the ulcervessel; 3: Ulcer of posthemostasis; 4: Bleeding of just anal position of esophagogastric junction; 5: Hemostasis with Heater probe was performed to the bleeding; 6: Tear of posthemostasis.

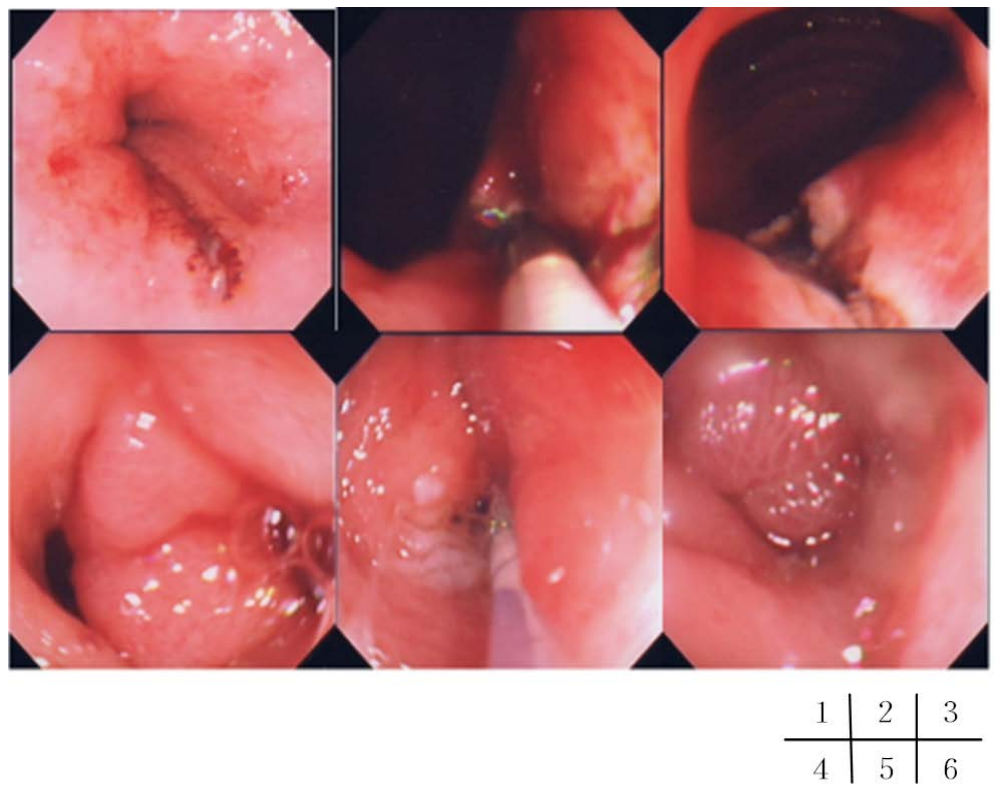

Figure 2. Upper gastrointestinal endoscopy of the case 2. 1: Bleeding of just anal position of esophagogastric junction; 2: Hemostasis with Heater probe was performed to the bleeding; 3: Tear of posthemostasis Ulcer of gastric antrum; 4: Ulcer bleeding of gastric antrum; 5: Hemostasis with Heater probe was performed to the ulcervessel; 6 : Ulcer of posthemostasis.

Case 6: A 57-year-old man was under observation for cirrhosis. An emergency endoscopy for hematemesis revealed a ruptured varicose vein at the esophagogastric mucosal junction. Therefore, EO sclerotherapy was performed, and MWS was observed after treatment necessitating a further hemostatic procedure. The patient was hospitalized, there was no recurrent bleeding, and he recovered well (Figure 6). 


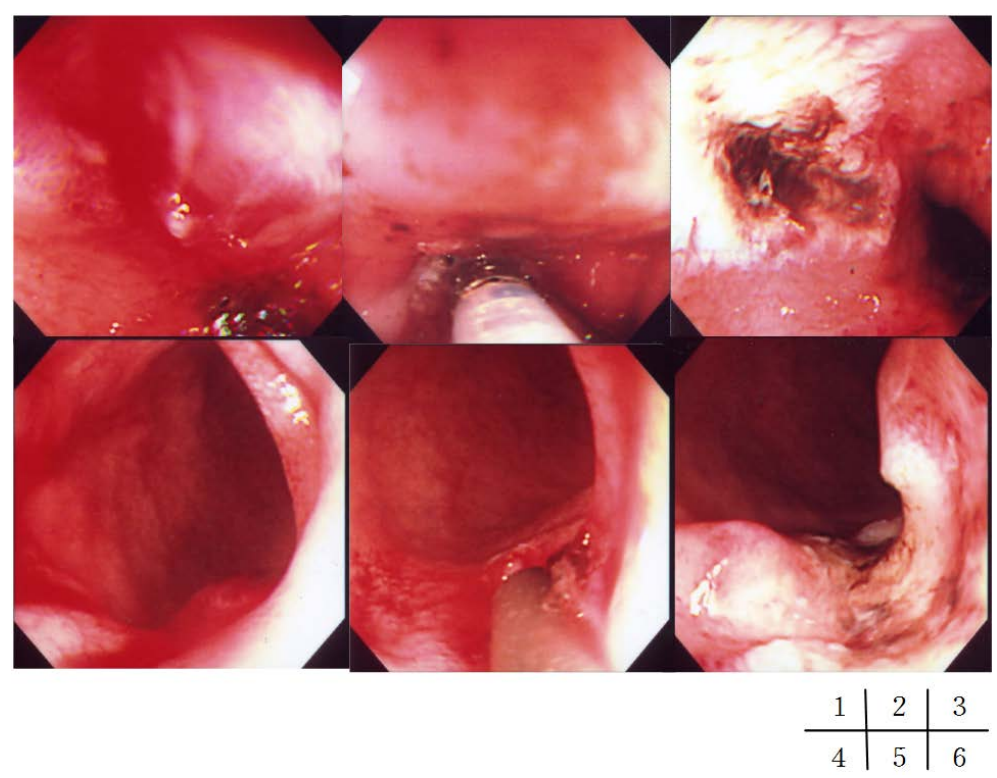

Figure 3. Upper gastrointestinal endoscopy of the case 3. 1: Bleeding of just anal position of esophagogastric mucosal junction; 2: Hemostasis with Heater probe was performed to the bleeding; 3: Tear of post hemostasis; 4: Bleeding of duodenal SDA; 5: Hemostasis with Heater probe was performed to the bleeding; 6: Ulcer of posthemostasis.

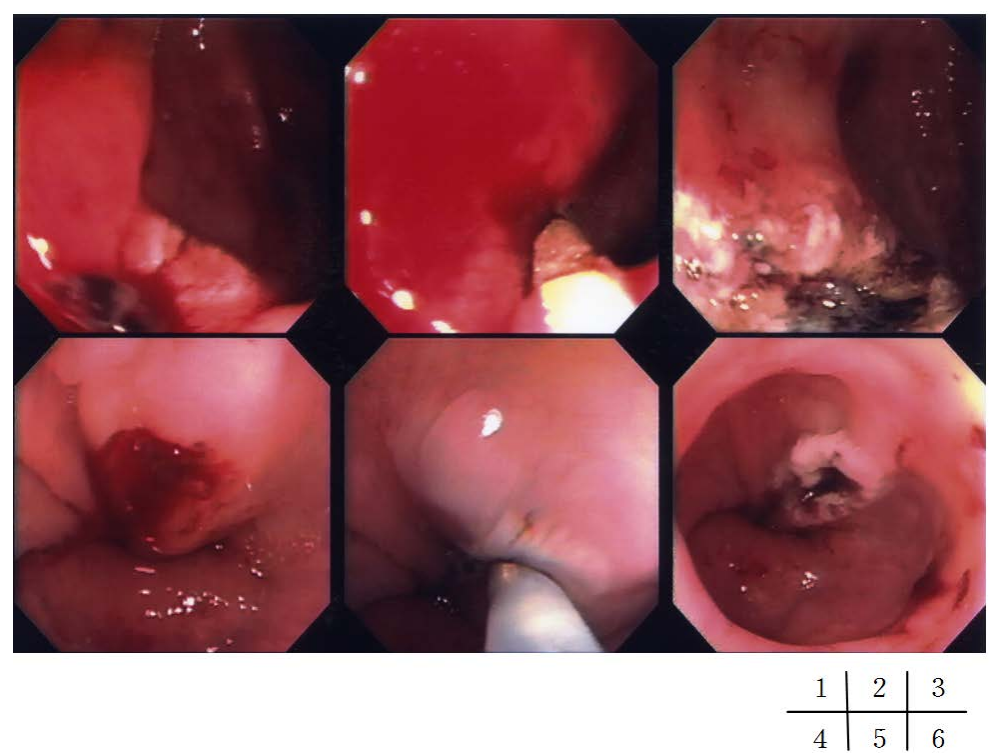

Figure 4. Upper gastrointestinal endoscopy of the case 4. 1: Bleeding of duodenal SDA; 2: Hemostasis with Heater probe was performed to the bleeding; 3: Ulcer of posthemostasis; 4: Bleeding of just anal position of esophagogastric mucosal junction; 5: Hemostasis with Heater probe was performed to the bleeding; 6: Tear of post hemostasis.

\section{Discussion}

The term MWS was coined in 1929 when Mallory and Weiss reported a case in which hematemesis occurred in a patient who repeatedly vomited after drinking alcohol [1]. The bleeding in MWS originates from lacerations near the esophagogastric mucosal junction. These lacerations develop during the rapid increase and restoration of pressure within the stomach due to vomiting, which causes esophageal invagination of the stomach near the 

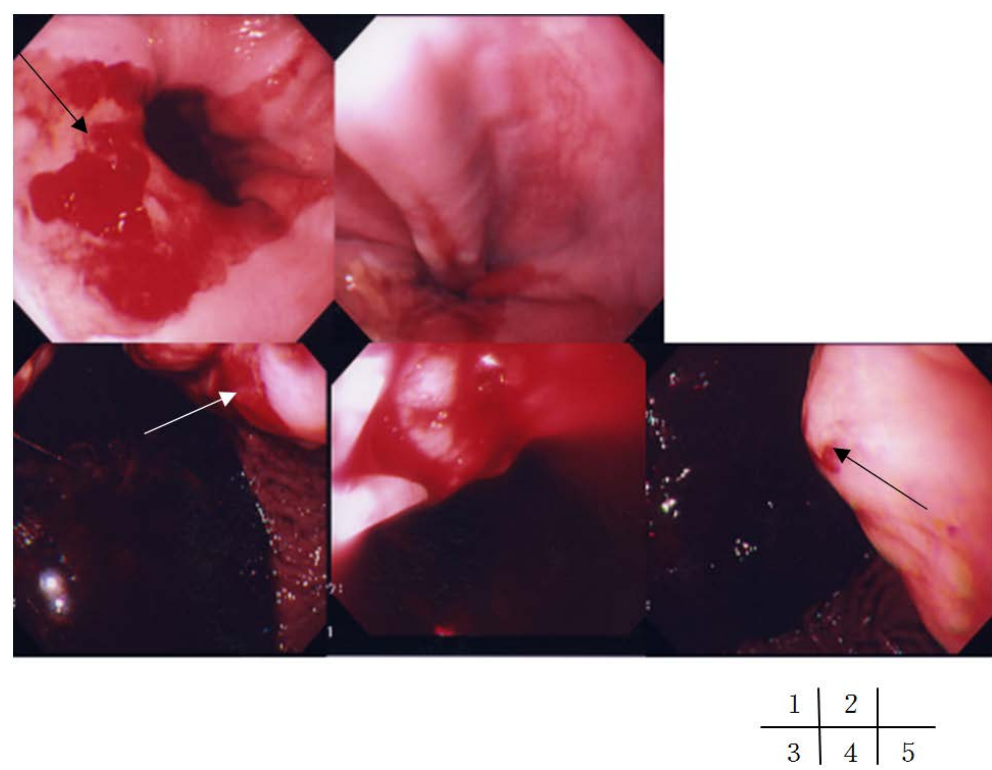

Figure 5. Upper gastrointestinal endoscopy of the case 5. 1: Bleeding of just anal position of esophagogastric mucosal junction (arrow); 2: Tear of posthemostasis; 3: Bleeding of esophageal varix (arrow); 4: Hemostasis with EIS; 5: Varix of posthemostasis (arrow is injection point).

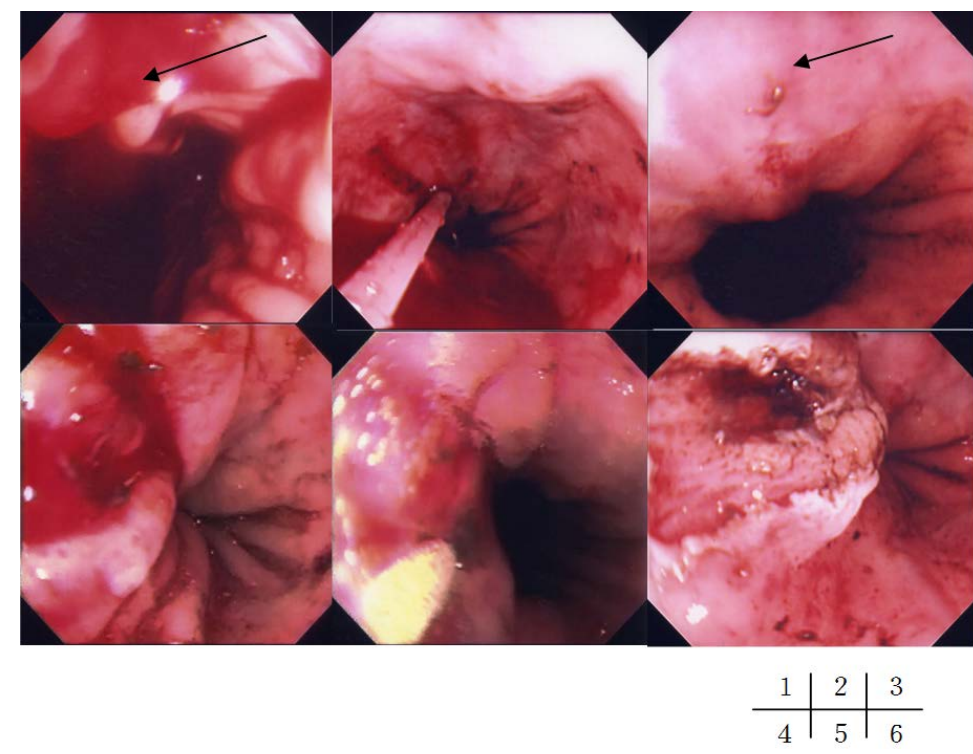

Figure 6. Upper gastrointestinal endoscopy of the case 6. 1: Bleeding of esophageal varix (arrow); 2: Hemostasis with EIS; 3: Varix of posthemostasis (arrow is injection point); 4: Bleeding of just anal position of esophagogastric mucosal junction; 5: Hemostasis with Heater probe was performed to the bleeding; 6: Tear of posthemostasis.

junction (mushrooming) [2] and the area near the junction to rapidly dilate.

The etiology of MWS has generally focused on its association with heavy drinking. However, MWS can also develop due to endoscopic examination and treatment [3] [4], hyperemesis gravidarum, or preliminary treatments for large bowel endoscopy, where these are associated with nausea, vomiting, straining, coughing, abdominal injury, or epileptic seizures [5]. If all layers of the esophageal wall tear and create an opening to the mediastinum, the condition manifests differently as an idiopathic esophageal rupture (Boerhaave's syndrome) [6]. 
In particular, MWS primarily manifests as gastrointestinal bleeding, whereas idiopathic esophageal rupture manifests as inflammation that extends to the mediastinum or the thoracic cavity. Zeifer classified lacerations near the esophagogastric mucosal junction into Group I (esophageal type), Group II (gastric type), and Group III (gastric and esophageal type) [7]. The most common of these is Group II, and all cases outlined in this report were also gastric.

MWS is a primary cause of upper gastrointestinal bleeding. It is known to develop in patients who have undergone endoscopic treatment for bleeding ulcers and ruptured varicose veins. The number of upper gastrointestinal bleeding cases examined at our facility is shown in Table 1. As described above, vomiting can arise due to causes other than excessive drinking. Notably, MWS can be induced by hematemesis during upper gastrointestinal bleeding or stimulation of the vomiting reflex during upper gastrointestinal endoscopy [8] [9]. This can confuse searches for the cause of gastrointestinal bleeding and requires additional treatment.

The primary lesions were gastric ulcers, duodenal ulcers, and esophageal varices. For each primary lesion, there was a case where MWS was simultaneously treated and a case where MWS was treated first. In the latter scenario, the primary lesion was overlooked during initial endoscopy, but was successfully treated after recurrent bleeding. There are possible explanations for these missed diagnoses during initial endoscopy. For example, the gastric ulcer may have been hidden in the deformed antrum (such as the case 2) and the duodenal ulcer could have been directly under the superior duodenal angle (such as the case 3), making each difficult to observe. However, it is possible that insufficient effort was made to identify a source of bleeding other than MWS in the case of the ruptured varicose vein (such as the case 5). Moreover, we cannot exclude the possibility that the varicose vein ruptured and began bleeding only after MWS treatment. Furthermore, in cases where the patient was simultaneously treated for MWS, it is likely that MWS occurred during endoscopic treatment because it was not confirmed when the endoscope was inserted. In addition, we almost always perform a second look endoscopy at our facility [10]. Among the three cases where the primary lesion was not identified during initial endoscopy, we failed to find the two ulcers during the second look endoscopy, and the varicose vein started to bleed before endoscopy could be performed.

When MWS is detected, it is prudent both to treat MWS as necessary and to search for other potential sources of bleeding. As evidenced above, it is also necessary to thoroughly examine difficult-to-observe areas such as the posterior wall of the duodenal bulb and the area directly below the superior duodenal angle. To avoid overlooking pathology, second look endoscopy is crucial when the stomach cannot be sufficiently observed due to blood in the primary procedure. Furthermore, if the patient has cirrhosis, it is important to carefully examine the area near the esophagogastric mucosal junction to determine if there is a ruptured varicose vein.

\section{Conclusion}

MWS is a common disease; however, due to its mechanism of onset, it can develop secondary to either another

\begin{tabular}{cc|}
\hline Table 1. The cases of upper gastrointestinal bleeding $(2002-2011)$. \\
\hline Disease & Cases \\
\hline Gastric ulcer & 440 \\
Varices & 340 \\
Duodenal ulcer & 190 \\
Esophageal bleeding & 127 \\
Mallory-Weiss syndrome & 79 \\
AGML & 75 \\
Others & 127 \\
Total & 1378 \\
\hline
\end{tabular}

AGML: acute gastric mucosal legion. 
disease that causes bleeding or to endoscopic procedures to manage the primary disease. This fact should be considered when managing a patient with MWS, and care must be taken during endoscopies to ensure that no pathology is overlooked.

\section{Conflict of Interests}

Authors declare no conflict of interests for this article.

\section{References}

[1] Mallory, G.K. and Weiss, S. (1929) Hemorrhages from Lacerations of the Cardiac of the Stomach Due to Vomiting. American Journal of the Medical Sciences, 178, 506-515. http://dx.doi.org/10.1097/00000441-192910000-00005

[2] Sugawa, C. and Masuyama, H. (1986) Mallory-Weiss Syndrome-A Changing Clinical Picture of 310 Patients. Japanese Journal of Gastroenterology, 83, 619-624.

[3] Kitagawa, T., Takano, H., Soma, M., Muto, E. and Takeda, S. (1994) Clinical Study of Mallory-Weiss Syndrome in the Aged Patients over 75 Year-Mainly Five Cases Induced by the Endoscopic Examination. Japanese Journal of Geriatrics, 31, 374-379. http://dx.doi.org/10.3143/geriatrics.31.374

[4] Kasamaki, S., Okada, T. and Okada, S. (2010) Nine Cases of Gastric Mucosal Injury Due to Mallory-Weiss Syndrome during Percutaneous Endoscopic Gastrostomy (PEG). Gastroenterological Endoscopy, 52, 1691-1697.

[5] Nishida, Y., Hamaji, M., Sakabuchi, H., Miyazaki, T., Kuhara, A., Ishida, M. and Ito, T. (1999) Mallory-Weiss Syndrome with Colonic Lavage-A Case Report. The Japanese Journal of Gastroenterological Surgery, 32, 997-1001. http://dx.doi.org/10.5833/jigs.32.997

[6] Inoue, Y. (1994) Mallory-Weiss Syndrome. Japanese Journal of Acute Medicine, 18, 1330-1332.

[7] Zeifer, H.D. (1961) Mallory-Weiss Syndrome. Annals of Surgery, 154, 956-960.

[8] Watts, H.D. (1976) Mallory-Weiss Syndrome Occurring as a Complication of Endoscopy. Gastrointestinal Endoscopy, 22, 171. http://dx.doi.org/10.1016/S0016-5107(76)73737-4

[9] Baker, R.W., Spiro, A.H. and Trnka, Y.M. (1982) Mallory-Weiss Tear Complicating Upper Endoscopy: Case Reports and Review of the Literature. Gastroenterology, 82, 140-142.

[10] Inoue, Y., Fujino, Y., Onodera, M., Kikuchi, S., Kojika, M. and Endo, S. (2012) Emergency Endoscopy for Upper Gastrointestinal and Non-Variceal Bleeding. Japanese Journal of Gastroenterology, 55, 545-552. 
Scientific Research Publishing (SCIRP) is one of the largest Open Access journal publishers. It is currently publishing more than 200 open access, online, peer-reviewed journals covering a wide range of academic disciplines. SCIRP serves the worldwide academic communities and contributes to the progress and application of science with its publication.

Other selected journals from SCIRP are listed as below. Submit your manuscript to us via either submit@scirp.org or Online Submission Portal.
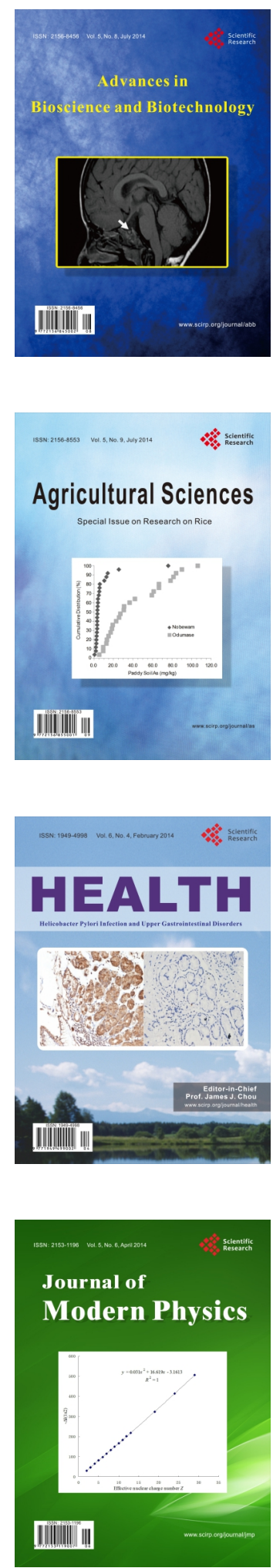
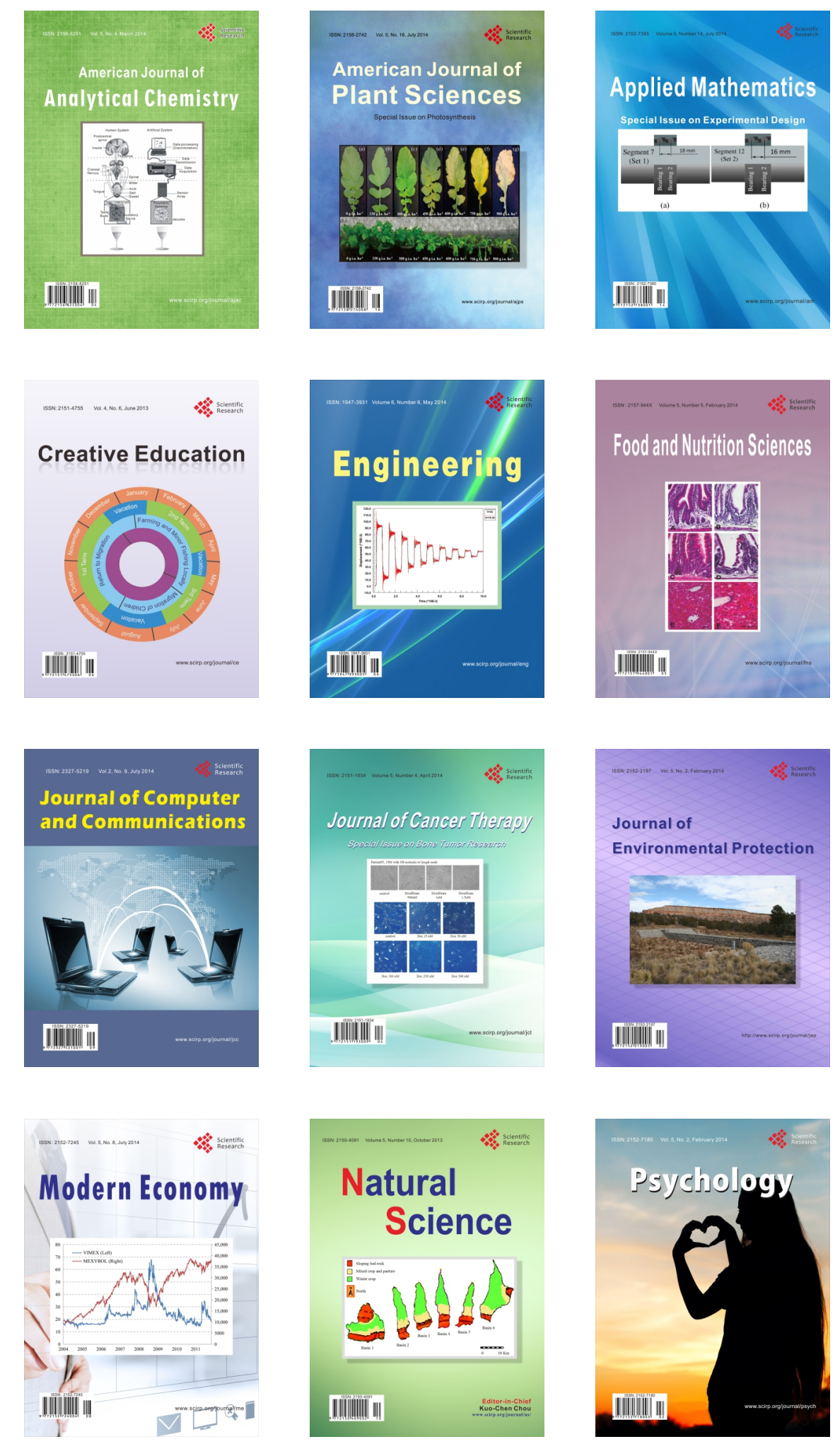